\title{
Il carcinoma familiare non midollare della tiroide non sindromico
}

\author{
Marco Capezzone $^{1} \cdot$ Maria Grazia Castagna ${ }^{1}$
}

Accettato: 4 marzo 2021 / Pubblicato online: 1 settembre 2021

(c) The Author(s) 2021

Sommario Il carcinoma non midollare della tiroide (non medullary thyroid cancer, NMTC) è generalmente sporadico ma può presentarsi in forma familiare (familial non medullary thyroid cancer, FNMTC) in circa il $10 \%$ dei casi. Negli anni si sono accumulate evidenze a favore di una predisposizione genetica ereditaria del FNMTC, come la scoperta di alcuni loci di suscettibilità, la presenza di alterazioni molecolari a carico del complesso telomero-telomerasi e l'evidenza di polimorfismi a singolo nucleotide (SNPs) associati statisticamente al rischio di sviluppare la malattia. Molti studi clinici concordano nell'attribuire al FNMTC un fenotipo più aggressivo rispetto alla controparte sporadica, supportando l'ipotesi che esso rappresenti una entità $a$ sé, clinicamente distinta dalla forma sporadica. Sebbene la presenza di possibile familiarità per carcinoma tiroideo debba essere sempre valutata attraverso un'accurata anamnesi familiare, le attuali linee guida non si esprimono a favore o contro lo screening ecografico nei pazienti con FNMTC non-sindromico. Tuttavia, alla luce delle più attuali conoscenze, sembrerebbe ragionevole raccomandare uno screening ecografico almeno nelle famiglie con tre o più membri affetti.

Parole chiave Carcinoma familiare della tiroide non midollare - Carcinoma non midollare non sindromico . Carcinoma non midollare sindromico

Proposto da Maria Grazia Castagna.

Informazioni Supplementari La versione online contiene materiale supplementare disponibile su

https://doi.org/10.1007/s40619-021-00950-0.

M.G. Castagna

mariagrazia.castagna@unisi.it

1 Dipartimento di Scienze Mediche, Chirurgiche e Neuroscienze, Struttura Complessa di Endocrinologia e Malattie Metaboliche, Università di Siena, Siena, Italia

\section{Introduzione}

I carcinomi familiari, a differenza di quelli ereditari causati da una identificata mutazione genetica, sono definiti dalla presenza della neoplasia in più membri di una stessa famiglia, in assenza di una mutazione germinale nota. Tutte le forme di neoplasie umane possono decorrere in forma familiare compresi i carcinomi, i sarcomi, i tumori del cervello e degli organi ematopoietici. Anche il carcinoma non midollare della tiroide (non medullary thyroid cancer, NMTC), nonostante sia in prevalenza sporadico, può essere familiare in circa il $10 \%$ dei casi [1]. Lo spettro di presentazione di tali forme familiari è eterogeneo, ed esprime almeno tre fenotipi: 1) una forma familiare sindromica, particolarmente rara, in cui il carcinoma tiroideo si associa a malattie non tiroidee come la sindrome di Gardner, la sindrome di Cowden, la Sindrome di Werner o il complesso di Carney e in cui un gene responsabile è stato identificato (Tabella 1); 2) un secondo fenotipo, anch'esso molto raro, in cui il cancro tiroideo familiare si associa ad un istotipo particolare con loci di suscettibilità ben definiti (Tabella 2); 3) la forma più frequente, quella in cui il FNMTC rappresenta l'unica manifestazione clinica in assenza di geni candidati (Fig. 1).

\section{Definizione}

Tradizionalmente il FNMTC è definito dalla presenza del tumore in due o più familiari di primo grado [2]. Tuttavia, tale definizione è stata messa in discussione, in quanto la presenza di due soli familiari potrebbe rappresentare, secondo uno studio di simulazione statistica, l'associazione casuale di due tumori sporadici in circa il $66 \%$ dei casi. Se i familiari affetti sono invece tre, il rischio di un' associazione casuale si riduce a meno del 5\% [3]. Nella pratica clinica, tuttavia, 
Tabella 1 Sindromi genetiche associate al carcinoma tiroideo non-midollare

\begin{tabular}{lll}
\hline Sindrome familiare & Gene & Tumore tiroideo (incidenza \%) \\
\hline Poliposi adenomatosa familiare & APC & Carcinoma papillare con pattern cribriforme (2-12\%) \\
Sindrome di Cowden & PTEN & Carcinoma papillare o follicolare (> 10\%) \\
Complesso di Carney tipo 1 & PRKARI & Carcinoma follicolare e papillare (4-60\%) \\
Sindrome di Werner & WRN & Carcinoma papillare, follicolare o anaplastico (18\%) \\
\hline
\end{tabular}

Tabella 2 Loci di suscettibilità associati al carcinoma familiare nonmidollare della tiroide (FNMTC)

\begin{tabular}{lll}
\hline Tumore & Localizzazione cromosomica & Numero OMIM \\
\hline PTC/PRN & $1 \mathrm{q} 21$ & 606240 \\
PTC & $2 \mathrm{q} 21$ & 606383 \\
TCO & $19 \mathrm{p} 13.2$ & 606240 \\
\hline
\end{tabular}

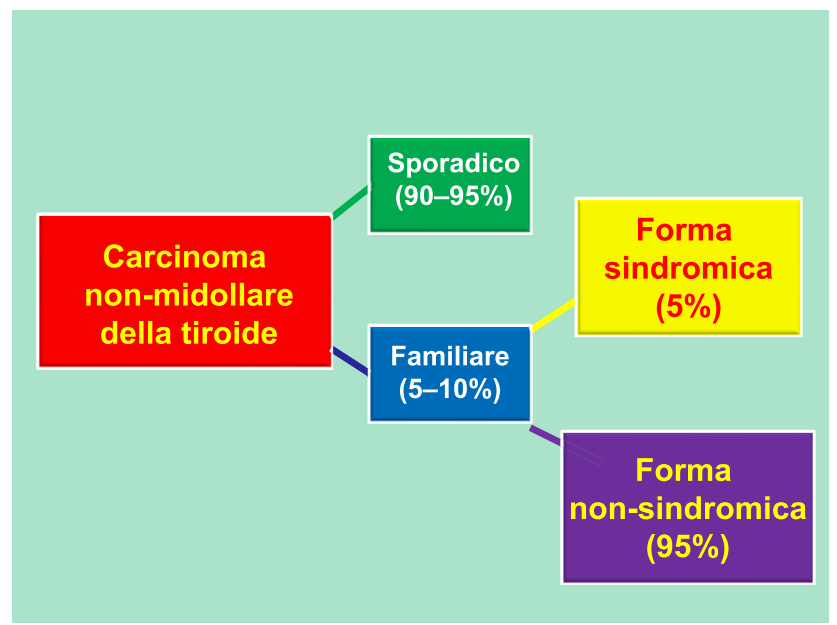

Fig. 1 Incidenza del carcinoma sporadico e familiare non midollare della tiroide (NMTC)

le famiglie FNMTC costituite da tre o più membri affetti, rappresentano solo il 5\% dei casi [4].

Recentemente è stato suggerito di utilizzare, nelle famiglie con due soli affetti, l'età alla diagnosi come criterio differenziale. Secondo questo studio, se il probando e il familiare affetto hanno entrambi, al momento della diagnosi della malattia, un'età inferiore ai 45 anni, il rischio di un' associazione casuale potrebbe essere minimo dal momento che le forme familiari sono generalmente caratterizzate da una precoce età di insorgenza e dal fenomeno dell'anticipazione genica [5].

\section{Aspetti genetici}

L'ipotesi che il FNMTC sia a trasmissione ereditaria è stato suggerito da studi epidemiologici e dall'analisi di alcuni registri nazionali del cancro, come quelli svedesi e norvege- si, che hanno evidenziato come, tra tutti i tipi di cancro, il carcinoma non-midollare della tiroide era quello con un rischio significativamente più alto di svilupparsi nei parenti di primo grado di un soggetto affetto da tale patologia rispetto alla popolazione generale [6,7].

Numerose casistiche riportano la presenza di forme familiari in percentuali variabili tra 5 e $10 \%$. Su questa base, è possibile che esista una predisposizione genetica ereditaria trasmessa con carattere autosomico-dominante a scarsa penetranza [8].

Alcune regioni genomiche considerate come loci predisponenti allo sviluppo di FNMTC sono state riportate in vari studi. Il primo locus (denominato thyroid carcinoma with oxyphilia, TCO) è stato localizzato in posizione 19p13.2 in una singola famiglia francese con una rara forma di tumore familiare della tiroide a cellule ossifile [9]. Un altro locus è stato identificato in posizione 1q21 in una famiglia statunitense affetta sia da FNMTC che da neoplasia papillare del rene (papillary thyroid carcinoma and papillary renal neoplasia, fPTC/PRN) [10]. Un ulteriore locus di suscettibilità (non-medullary thyroid carcinoma 1 , NMTC1) è stato identificato sul cromosoma $2 \mathrm{q} 21$ in una grande famiglia proveniente dalla Tasmania affetta da un'alta frequenza di carcinoma papillare variante follicolare della tiroide [11].

Per quanto riguarda il FNMTC isolato, non è stato trovato alcun locus di suscettibilità e non sono state dimostrate mutazioni germinali a carico degli oncogeni noti. Le classiche alterazioni genetiche più comunemente descritte nel NMTC a livello somatico (le mutazioni puntiformi di BRAF e RAS e i riarrangiamenti di RET/PTC, PPARgamma e TRK) non sono state dimostrate a livello germinale nel FNMTC. Non è ancora stato chiarito al momento se ci sia una differenza nella prevalenza delle mutazioni somatiche a carico di oncogeni noti tra pazienti con FNMTC e pazienti con NMTC sporadico.

Fino ad oggi sono stati identificati circa 19 geni di suscettibilità al FNMTC (Tabella 3). Nel 2015, Gara e collaboratori hanno pubblicato sul New England Journal of Medicine la presenza di una variante non sinonima G534E (rs7080536, allele A) del gene HABP2 come causa dello sviluppo del FNMTC. Molti studi successivi hanno tuttavia fallito nel confermare i risultati dello studio del NEJM, concludendo che HABP2 G534E è una variante a bassa o moderata frequenza e non è associata con il rischio di FNMTC, indipendentemente dal tipo istologico [12]. 
Tabella 3 Geni e loci di suscettibilità associati al carcinoma familiare non-midollare della tiroide (FNMTC) non-sindromico

\begin{tabular}{ll}
\hline Locus cromosomico & Gene \\
\hline $9 \mathrm{q} 22.23$ & FOXE1 \\
$14 \mathrm{q} 13.3$ & NKX2-1 \\
$12 \mathrm{q} 14.2$ & SRGAP1 \\
$15 \mathrm{q} 23$ & MAP2K5 \\
$20 \mathrm{p} 12.3$ & PLCB1 \\
$10 \mathrm{q} 25.3$ & HABP2 \\
$1 \mathrm{q} 41$ & BROX \\
$7 \mathrm{q} 31.33$ & POT1 \\
$19 \mathrm{p} 13.33$ & NOP53 \\
$22 \mathrm{q} 12.1$ & CHEK2 \\
$19 \mathrm{p} 13.11$ & NDUFA13 \\
$19 \mathrm{p} 13.2$ & TIMM44 \\
$4 \mathrm{q} 21.21$ & ANXA3 \\
$12 \mathrm{q} 22$ & NTN4 \\
$14 \mathrm{q} 32.13$ & SERPINA1 \\
$17 \mathrm{q} 21.2$ & FKBP10 \\
$1 \mathrm{p} 36.31$ & PLEKHG5 \\
$17 \mathrm{p} 13.2$ & P2RX5 \\
$6 \mathrm{p} 21.33$ & SAPCD1 \\
\hline
\end{tabular}

Recentemente, studi di associazione genome-wide (GWAS) compiuti su un ampio numero di pazienti e di casicontrollo appartenenti a diversi tipi di popolazioni hanno condotto all'identificazione di ulteriori loci di suscettibilità per il FNMTC. Tra questi, i più riproducibili sono risultati: rs966423 (DIRC3), rs2439302 (NRG1), rs965513 (PTCSC2/FOXE1), rs944289 (PTCSC3/NKX2-1) e rs116909374 (MBIP1) [13].

Sebbene l'esatto meccanismo biologico degli effetti di tutte le varianti non sia stato completamente compreso, l'elevata significatività statistica dei segnali di associazione implica inequivocabilmente il loro coinvolgimento nella carcinogenesi tiroidea. I risultati degli studi sugli SNPs sono consistenti con un modello multifattoriale e poligenico di suscettibilità al carcinoma tiroideo.

\section{Aspetti clinici}

È controverso in letteratura se il FNMTC abbia un comportamento clinico diverso rispetto al carcinoma differenziato sporadico. Tuttavia, la maggioranza degli studi sottolineano come il FNMTC abbia generalmente un fenotipo più aggressivo alla diagnosi [14-17]. Le caratteristiche cliniche che vengono generalmente associate al FNMTC sono una più giovane età alla diagnosi, una maggior frequenza di multicentricità e bilateralità della neoplasia e un maggior tasso di metastasi linfonodali. La più giovane età alla diagnosi nell'FNMTC è stata da alcuni autori messa in relazione con la presenza di un possibile "effetto screening". Infatti, la diagnosi di tumore tiroideo nel probando potrebbe indurre a effettuare una valutazione morfo-funzionale tiroidea agli altri membri della stessa famiglia, portando così a una diagnosi precoce del tumore tiroideo. Tuttavia, uno studio italiano condotto su 20 famiglie affette da FNMTC, nel quale sono state confrontate le caratteristiche cliniche del FNMTC tra prima e seconda generazione, sottolineava come la precoce età di insorgenza della malattia nel FNMTC potesse essere spiegata con il cosiddetto "fenomeno dell' anticipazione", in cui le generazioni successive alla prima sviluppavano la malattia precocemente $\mathrm{e}$ in forma più aggressiva rispetto a quelle precedenti [15].

Il rilievo della maggiore multicentricità e bilateralità della neoplasia nei pazienti con FNMTC è documentato nella larga maggioranza degli studi presenti in letteratura e conferma l'osservazione riportata anche per altri tipi di cancro, secondo la quale la multifocalità, specialmente se associata a un'età del paziente relativamente giovane, rappresenta una caratteristica peculiare dei carcinomi di tipo familiare. In particolare, uno studio condotto su un'ampia coorte di pazienti dimostrava come, nei pazienti affetti da FNMTC, il tumore era più spesso multicentrico e maggiormente associato con la presenza di metastasi linfonodali e di patologie tiroidee benigne [16]. Uno studio più recente contribuiva a rafforzare il concetto che una storia familiare di carcinoma differenziato tiroideo possa rappresentare un fattore di rischio per una maggiore aggressività della neoplasia, dimostrando come nei pazienti con FNMTC fosse presente una maggiore multicentricità e invasione extraghiandolare della neoplasia e un più alto numero di metastasi linfonodali al momento dell'intervento chirurgico, rispetto ai pazienti con cancro tiroideo sporadico [17].

Un aspetto ancora oggi molto discusso riguarda il ruolo prognostico della familiarità nei pazienti affetti da NMTC. Sebbene ci sia sufficiente consenso sul fatto che il tasso di mortalità non sia differente tra le forme sporadiche e le forme familiari, controverso è ancora l'impatto della familiarità sul rischio di recidiva di malattia in corso di follow-up. Uno studio [18] compiuto su una casistica di circa 6.000 pazienti affetti da FNMTC, di cui 273 (4,5\%) classificati come FNMTC, non mostrava differenze riguardo i tassi di sopravvivenza di malattia tra i due gruppi, mentre riportava un maggior tasso di recidiva di malattia loco-regionale a 30 anni nel FNMTC (40\% per gli FNMTC rispetto al 20\% dei NMTC sporadici). Un più recente lavoro ha invece documentato come, a fronte di un maggior tasso di persistenza di malattia dopo il trattamento iniziale $(39,5$ vs $28,6 \%$, $p=0,04$ ), alla fine del follow-up (mediana di follow-up: 9 anni) il tasso di remissione clinica di malattia non era differente tra pazienti con carcinoma papillare sporadico e familiare $(79,3$ vs $84,3 \%, p=0,26)$ [17]. I risultati di questo 
Tabella 4 Metanalisi sull'incidenza e sul fenotipo del carcinoma papillare familiare tiroideo (CPFT) rispetto al carcinoma papillare tiroideo sporadico

\begin{tabular}{lllll}
\hline Autore & Anno & N. pazienti & Incidenza del CPFT $(\%)$ & $\begin{array}{l}\text { Fenotipo CPFT } v s \\
\text { CPT sporadico }\end{array}$ \\
\hline Stoffer & 1986 & $14 / 226$ & 6,2 & Più aggressivo \\
Hrafnkelsson & 1989 & $24 / 383$ & 6,3 & Più aggressivo \\
Grossman & 1995 & $14 / 289$ & 4,8 & Più aggressivo \\
Loh & 1997 & $178^{*}(* 15$ serie $)$ & $2,5-6,3$ & Non differenze \\
Kraimps & 1997 & $11 / 105$ & 10,5 & Più aggressivo \\
Uchino & 2002 & $258 / 6458$ & 4,0 & Più aggressivo \\
Maxwell & 2004 & $24 / 543$ & 4,4 & Non differenze \\
Capezzone & 2008 & $34 / 300$ & 11,3 & Più aggressivo \\
Ito & 2009 & $273 / 6015$ & 4,5 & Più aggressivo \\
Robenshtok & 2011 & $67 / 375$ & 17,8 & Non differenze \\
Park & 2012 & 13056 & 9,6 & Più aggressivo \\
Mazeh & 2012 & $37 / 358$ & 10,0 & Più aggressivo \\
\hline
\end{tabular}

studio suggeriscono che, sebbene il FNMTC sia più aggressivo della controparte sporadica, se adeguatamente trattato (trattamento aggiuntivo durante il follow-up quando necessario), ha un tasso di remissione clinica non differente da quello osservato nella forma sporadica. Le evidenze scientifiche di una maggiore estensione di malattia alla diagnosi e di una peggiore risposta al trattamento iniziale non devono, tuttavia, portare a una gestione terapeutica e di follow-up più aggressiva generalizzata nella forma familiare di NMTC. Un approccio terapeutico e di follow-up personalizzato dovrebbe essere il percorso da seguire nel paziente affetto da FNMTC.

La Tabella 4 riporta schematicamente l'incidenza e il fenotipo del carcinoma papillare familiare tiroideo (CPFT) rispetto al carcinoma papillare tiroideo sporadico.

\section{La questione dello screening}

Mentre lo screening per il NMTC sporadico è considerato non necessario o addirittura potenzialmente dannoso, alcuni autori hanno proposto uno screening annuale nelle famiglie con FNMTC. I fautori dello screening giustificano il loro punto di vista riportando gli alti tassi di familiarità del carcinoma tiroideo differenziato e la peggiore presentazione fenotipica ed esito dei pazienti FNMTC rispetto a quelli con forma sporadica. Un recente studio prospettico con screening annuale di individui provenienti da famiglie con FNMTC ha riportato una prevalenza di cancro della tiroide mediante screening ecografico del 4,6\% negli individui provenienti da famiglie con due membri affetti (simile alla prevalenza stimata nella popolazione generale di $4,5 \%$ ) e del $22,7 \%$ degli individui provenienti da famiglie con tre o più pazienti affetti [19]. Analoghi risultati sono stati riportati in uno studio prospettico [20] che ha valutato prevalentemente famiglie con solo due membri affetti, riportando il 5,5\% di prevalenza di cancro alla tiroide nei familiari di soggetti con FNMTC.

Gli interventi di screening sono generalmente raccomandati quando la diagnosi e il trattamento terapeutico precoce conducono a un miglioramento della qualità di vita e a una riduzione del tasso di mortalità, ma nessuno studio ha riportato questi risultati nel caso del carcinoma differenziato della tiroide. Le attuali Linee guida [21] non si esprimono a favore né contro lo screening ecografico nei pazienti con FNMTC non-sindromico. Tuttavia, alla luce dei più recenti dati di letteratura sembrerebbe ragionevole raccomandare uno screening ecografico annuale nelle famiglie con tre o più affetti. Nelle famiglie con due soli affetti i dati sono insufficienti per raccomandare uno screening ecografico annuale dato il rischio di un'associazione di tumori sporadici e il rischio di over-diagnosis e over-treatment.

\section{Conclusioni}

Sebbene al momento, contrariamente a quanto avviene nel carcinoma midollare della tiroide, non siano state documentate mutazioni geniche a livello germinale responsabili dell'insorgenza del carcinoma papillare familiare della tiroide, numerose evidenze sia di carattere genetico che clinico sembrerebbero confermare l'esistenza del FNMTC come una vera entità clinica distinta dal NMTC sporadico. Visto il fenotipo più aggressivo e una prognosi peggiore almeno nel breve termine, un approccio terapeutico e di follow-up personalizzato (trattamenti terapeutici aggiuntivo solo quando necessari) dovrebbe essere il percorso da seguire nel paziente affetto da FNMTC. In aggiunta, lo screening ecografico limitato alle famiglie con FNMTC in cui sono presento tre o più membri affetti, potrebbe garantire una diagnosi precoce e nel contempo evitare l'over-diagnosis e l'over-treatment 
che si avrebbe in caso di uno screening generalizzato dei familiari di pazienti con FNMTC.

Funding Note Open access funding provided by Università degli Studi di Siena within the CRUI-CARE Agreement.

Conflitto di interesse Gli autori Marco Capezzone e Maria Grazia Castagna dichiarano di non avere conflitti di interesse.

Consenso informato Lo studio presentato in questo articolo non ha richiesto sperimentazione umana.

Studi sugli animali Gli autori di questo articolo non hanno eseguito studi sugli animali.

Nota della casa editrice Springer Nature rimane neutrale in riguardo alle rivendicazioni giurisdizionali nelle mappe pubblicate e nelle affiliazioni istituzionali.

Open Access This article is licensed under a Creative Commons Attribution 4.0 International License, which permits use, sharing, adaptation, distribution and reproduction in any medium or format, as long as you give appropriate credit to the original author(s) and the source, provide a link to the Creative Commons licence, and indicate if changes were made. The images or other third party material in this article are included in the article's Creative Commons licence, unless indicated otherwise in a credit line to the material. If material is not included in the article's Creative Commons licence and your intended use is not permitted by statutory regulation or exceeds the permitted use, you will need to obtain permission directly from the copyright holder. To view a copy of this licence, visit http://creativecommons.org/licenses/by/4.0/.

\section{Bibliografia}

1. Capezzone M, Robenshtok E, Cantara S, Castagna MG (2021) Familial non-medullary thyroid cancer: a critical review. J Endocrinol Invest 44(5):943-950

2. Mazeh H, Sippel RS (2013) Familial nonmedullary thyroid carcinoma. Thyroid 23(9):1049-1056

3. Charkes ND (2006) On the prevalence of familial nonmedullary thyroid cancer in multiply affected kindreds. Thyroid 16(2):181-186

4. Nixon IJ, Suárez C, Simo R et al (2016) The impact of family history on non-medullary thyroid cancer. Eur J Surg Oncol 42(10): 1455-1463

5. Capezzone M, Sagnella A, Pilli T et al (2021) Role of age at diagnosis in defining potential familial nonmedullary thyroid cancer in kindreds with two affected members. J Clin Endocrinol Metab 106(2):e855-e865

6. Hemminki K, Vaittinen P (1999) Familial cancers in a nationwide family cancer database: age distribution and prevalence. Eur J Cancer 35(7):1109-1117
7. Frich L, Glattre E, Akslen LA (2001) Familial occurrence of nonmedullary thyroid cancer: a population-based study of 5673 firstdegree relatives of thyroid cancer patients from Norway. Cancer Epidemiol Biomark Prev 10(2):113-117

8. Sturgeon C, Clark OH (2005) Familial nonmedullary thyroid cancer. Thyroid 15(6):588-593

9. Canzian F, Amati P, Harach HR et al (1998) A gene predisposing to familial thyroid tumors with cell oxyphilia maps to chromosome 19p13.2. Am J Hum Genet 63(6):1743-1748

10. Malchoff CD, Sarfarazi M, Tendler B et al (2000) Papillary thyroid carcinoma associated with papillary renal neoplasia: genetic linkage analysis of a distinct heritable tumor syndrome. J Clin Endocrinol Metab 85(5):1758-1764

11. McKay JD, Lesueur F, Jonard L et al (2001) Localization of a susceptibility gene for familial nonmedullary thyroid carcinoma to chromosome 2q21. Am J Hum Genet 69(2):440-446

12. Gara SK, Jia L, Merino MJ et al (2015) Germline HABP2 mutation causing familial nonmedullary thyroid cancer. N Engl J Med 373(5):448-455

13. Miasaki FY, Fuziwara CS, Carvalho GA, Kimura ET (2020) Genetic mutations and variants in the susceptibility of familial non-medullary thyroid cancer. Genes (Basel) 11(11):1364

14. Robenshtok E, Tzvetov G, Grozinsky-Glasberg S et al (2011) Clinical characteristics and outcome of familial nonmedullary thyroid cancer: a retrospective controlled study. Thyroid 21(1):43-48

15. Capezzone M, Marchisotta S, Cantara S et al (2008) Familial nonmedullary thyroid carcinoma displays the features of clinical anticipation suggestive of a distinct biological entity. Endocr-Relat Cancer 15(4):1075-1081

16. Uchino S, Noguchi S, Kawamoto H et al (2002) Familial nonmedullary thyroid carcinoma characterized by multifocality and a high recurrence rate in a large study population. World J Surg 26(8):897-902

17. Mazeh H, Benavidez J, Poehls JL et al (2012) In patients with thyroid cancer of follicular cell origin, a family history of nonmedullary thyroid cancer in one first-degree relative is associated with more aggressive disease. Thyroid 22(1):3-8

18. Ito Y, Kakudo K, Hirokawa M et al (2009) Biological behavior and prognosis of familial papillary thyroid carcinoma. Surgery 145(1):100-105

19. Klubo-Gwiezdzinska J, Yang L, Merkel R et al (2017) Results of screening in familial non-medullary thyroid cancer. Thyroid 27(8):1017-1024

20. Ríos A, Rodríguez JM, Navas D et al (2016) Family screening in familial papillary carcinoma: the early detection of thyroid disease. Ann Surg Oncol 23(8):2564-2570

21. Haugen BR, Alexander EK, Bible KC et al (2016) 2015 American Thyroid Association management guidelines for adult patients with thyroid nodules and differentiated thyroid cancer: the American Thyroid Association Guidelines Task Force on Thyroid Nodules and Differentiated Thyroid Cancer. Thyroid 26(1):1-133 